\section{Xerophthalmia-a potential epidemic on our doorstep?}

S McLaughlin, J Welch, E MacDonald, S Mantry and K Ramaesh

\begin{abstract}
Purpose Xerophthalmia refers to the ocular manifestations associated with vitamin A deficiency, including xerosis, keratomalacia, nyctalopia and Bitot's spot. Hypovitaminosis $A$ is well-recognised in developing countries, but is rare in the developed world. Most cases in the latter relate to fat malabsorption. Conditions in which vitamin A metabolism or storage is deranged (chronic liver disease, including alcoholism) are also aetiologies.

We wanted to see whether this was common in our department.
\end{abstract}

Methods Oral vitamin A supplements were given to patients who presented with hypovitaminosis A.

Results All patients were found to have hypovitaminosis A on biochemical testing and responded dramatically to oral vitamin A supplementation, resulting in an improved final visual outcome.

Discussion This series demonstrates that prompt recognition and treatment of xerophthalmia can lead to rapid recovery and avert significant visual morbidity. The prevalence of xerophthalmia is likely to increase in the developed world largely owing to alcoholic liver disease. It is thought by some that we are on the verge of a potential epidemic. We hope that by increasing the profile of this important public health issue, we may be able to influence future prevalence of hypovitaminosis.

Eye (2014) 28, 621-623; doi:10.1038/eye.2014.17; published online 14 February 2014

Keywords: xerophthalmia; hypovitaminosis; alcoholism

\section{Introduction}

Xerophthalmia refers to the ocular manifestations associated with vitamin A deficiency. These include xerosis, keratomalacia, nyctalopia and Bitot's spots. Hypovitaminosis A is well recognised in developing countries, but is rare in the developed world. Most cases in the latter relate to inflammatory bowel disease, pancreatitis, upper gastrointestinal surgery and chronic liver disease (including alcoholism). ${ }^{1-4}$

We present a case series of patients who had hypovitaminosis $\mathrm{A}$ and responded markedly to oral vitamin A supplementation, resulting in an improved final visual outcome.

This series demonstrates that prompt recognition and treatment of xerophthalmia can lead to rapid recovery and avert significant visual morbidity.

\section{Case series}

\section{Case 1}

A 41-year-old man presented to eye casualty with bilateral red eyes and difficulty seeing at night. He admitted to excessive alcohol consumption (15+ units per day) and poor dietary intake.

His unaided distance visual acuities were 1/60 bilaterally. He had multiple, diffuse punctate epithelial erosions (PEE) on both corneas. Laboratory tests revealed deranged liver function and a markedly reduced vitamin A level of $<0.3 \mu \mathrm{mol} / 1$ (normal range 1.0-3.0 $\mu \mathrm{mol} / 1$ ) (see Figure 1).

The patient was given one dose of $100000 \mathrm{IU}$ of vitamin A intramuscularly and a regular high dose of vitamin A orally (4000 IU Vit A/D complex). Topical therapy comprised of intensive preservative-free ocular lubricants, G. chloramphenicol $0.5 \%$ and G. retinoic acid $0.1 \%$ twice daily.

He made rapid and progressive improvement and was discharged a week later with visual acuities of 6/12 in both eyes. His ocular surface was markedly clearer.
Tennent Institute of Ophthalmology, Gartnavel General Hospital, Glasgow, UK

Correspondence: S McLaughlin, Tennent Institute of Ophthalmology, Gartnavel General Hospital, Great Western Road, Glasgow G12 OYN, UK Tel: +44 (0)141 211 3000; Fax: +44 (0)1412111643. E-mail: sumonamclaughlin@ doctors.org.uk

Received: 12 July 2013 Accepted in revised form: 9 December 2013 Published online: 14 February 2014 


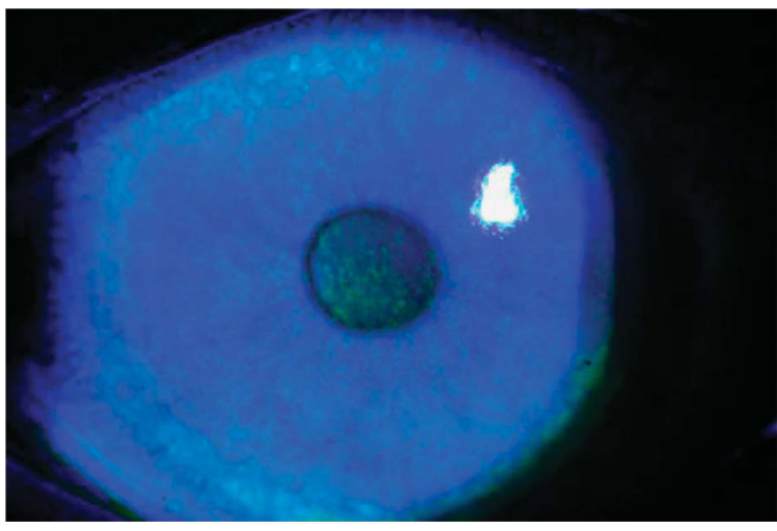

Figure 1 Corneal appearance of patient 1 on admission, with topical fluorescein instilled, demonstrating diffuse PEEs.

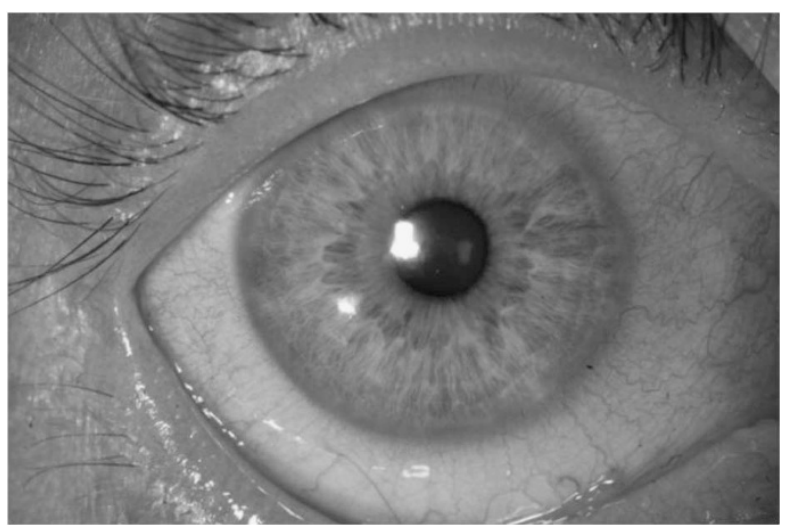

Figure 2 The right eye of patient 11 month after admission and treatment with vitamin A.

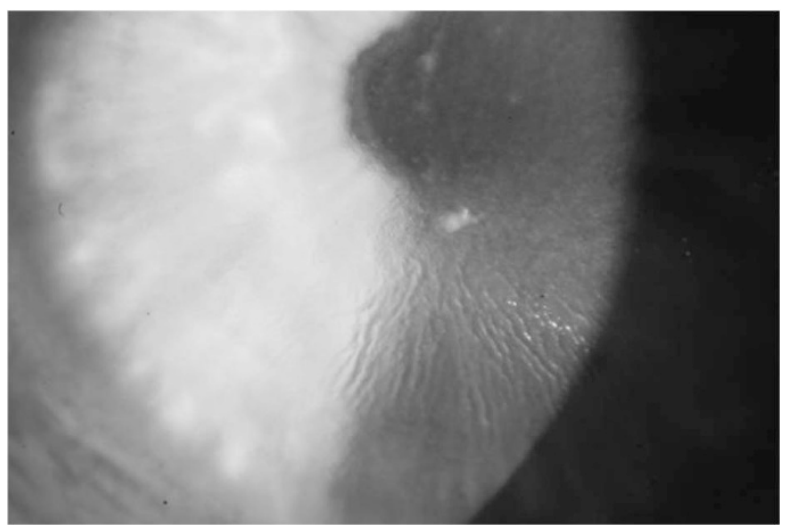

Figure 3 Appearance of the cornea of patient 3 on admission.

One month post admission, his best-corrected visual acuity was 6/6 in both eyes (see Figure 2).

\section{Case 2}

A 34-year-old man was referred to our corneal clinic with ocular pain, photophobia, persistent lacrimation, and reduced vision. He was a heavy smoker $(20+$ cigarettes per day) and admitted to very poor dietary intake.

His vitamin A level was $1.1 \mu \mathrm{mol} / 1$. He was commenced on oral vitamin A supplementation, and within a few weeks, his ocular surface and symptoms had improved. Six months after starting vitamin A supplements, his vitamin A levels had improved to $2.3 \mu \mathrm{mol} / 1$ and his ocular surface and symptoms were well controlled.

\section{Case 3}

A 49-year-old woman presented with bilateral blurred vision and ocular pain. Medical history included alcoholinduced pancreatitis. Visual acuities were $6 / 18$ bilaterally and she was diagnosed with bilateral hurricane keratopathy (see Figure 3).

Her vitamin A level was reduced, at $<0.3 \mu \mathrm{mol} / 1$, and hence she was started on oral vitamin A and folic acid supplements.

This provided some improvement in her symptoms, but it was in fact when she started topical retinoic acid $0.1 \%$ that she admitted to marked improvement. Three months after presentation, she was asymptomatic and her best-corrected visual acuities were $6 / 6+3$ in the right eye and 6/9 in the left eye.

\section{Discussion}

In 1995, the World Health Organization (WHO) estimated 254 million children to be vitamin A deficient worldwide and 2.8 million to have xerophthalmia. ${ }^{5}$

Vitamin A is an essential fat-soluble vitamin that is acquired through dietary intake. Normal levels are essential for maintenance of a healthy immune system, skin, ocular surface, and retina. ${ }^{6}$

Diagnosis of hypovitaminosis A is based on clinical signs, in the context of a suggestive past medical history. Serum vitamin A assays were performed in our patients to confirm the diagnosis. Conjunctival impression cytology and dark-adapted electroretinograms can also aid the diagnosis. ${ }^{7}$

The WHO advises treatment of severe xerophthalmia as a single oral dose of $200000 \mathrm{IU}$ vitamin A, followed by a further dose the following day and a final dose several weeks later. ${ }^{8}$

Topical treatment comprises preservative-free lubricants and antibiotics if secondary bacterial infection is present. G. retinoic acid $0.1 \%$ accelerates corneal healing, but may result in a more dense, vascularised scar. ${ }^{6}$

The goal of the WHO is the worldwide elimination of vitamin A deficiency and its tragic consequences, including blindness, disease, and premature death. ${ }^{5,9}$ We believe that it is underdiagnosed in the developed world. 
Conditions such as liver cirrhosis, malnutrition, and alcoholism allow for its presentation in the UK.

By raising the profile of xerophthalmia, clinicians will be able to recognise and treat keratomalacia at an earlier stage and commence timely vitamin A supplementation. This will undoubtedly improve visual morbidity significantly, as demonstrated in our case series and in others. ${ }^{10}$

In the UK, we must address this difficult public health issue of alcoholism as a priority. The effects of it are thought to be costing the NHS in excess of $£ 3$ billion per annum.

By highlighting alcohol abuse and subsequent hypovitaminosis A as an important cause of xerophthalmia, prompt recognition and treatment of keratomalacia will significantly reduce visual morbidity in these patients.

\section{Summary}

What was known before

- Hypovitaminosis A is well known in developing countries.

- Xerophthalmia has many causes in the developed world, such as gastric surgery and alcohol abuse.

- Xerophthalmia can cause significant visual morbidity and is relatively easy to treat.

\section{What this study adds}

- In the developed world, alcoholism is becoming a real problem.

- Always important to take a thorough social/dietary/ alcohol history in any patient with persistent dry eyes that are not responding to conventional treatment.

- Consider taking serum assays of vitamin levels, particularly vitamin A in this situation.

\section{Conflict of interest}

The authors declare no conflict of interest.

\section{References}

1 Roncone DP. Xerophthalmia secondary to alcohol-induced malnutrition. Optometry 2006; 77: 124-133.

2 Chae T, Foroozan R. Vitamin A deficiency in patients with a remote history of intestinal surgery. Br J Ophthalmol 2006; 90: 955-956.

3 Wamsley S, Patel SM, Wood MG, Mootha VV, Villalobos R, Albert DM et al. Advanced keratomalacia with descemetocele in an infant with cystic fibrosis. Arch Ophthalmol 2005; 123: 1012-1016.

4 Nicolai U, Rochels R. Bilateral severe keratomalacia after acute pancreatitis. Cornea 1993; 12: 171-173.

5 World Health Organization. The global prevalence of vitamin A deficiency. Micronutrient Deficiency Information System (MDIS) working paper 2: WHO/NUT/95 3. World Health Organization: Geneva, Switzerland, 1995.

6 Sommer A. Xerophthalmia and vitamin A status. Prog Retin Eye Res 1998; 17: 9-31.

7 Qureshi SH, Selva-Nayagam DN, Crompton JL. Hypovitaminosis A in metropolitan Adelaide. Clin Experimental Ophth 2000; 28: 62-64.

8 Paranjpe DR, Newton CJ, Pyott AEA. Nutritional disorders In: Krachmer JH, Mannis MJ, Holland EJ (eds). 3rd edn. Mosby Elsevier: Cornea, 2011, pp 721-732.

9 The Micronutrient Initiative. Progress in controlling vitamin A deficiency. The micronutrient initiative, the United Nations Children's Fund. Ottawa. Tulane University: Canada, 1998

10 Lin P, Fintelmann RE, Khalifa YM, Bailony MR, Jeng BH. ocular surface disease secondary to Vitamin A deficiency in the developed world: it still exists. Arch Ophthalmol 2011; 129(6): 798-804. 